was removed for histological purposes, and the state of the genital organs was ascertained as far as possible. In six animals which were examined at a later date again it was found that no compensatory hypertrophy had occurred. Fourteen of the animals were subjected to experiments with gonadotropic hormones ( $p$-factors, prolans) prepared in most cases from pregnancy urine by a method published previously, and in three cases anterior lobe grafts were given. It was found that within eleven to twenty-one days restitution of gonadic function occurred. Corpora lutea were found in the ovaries, and the uteri of these animals were found to be of functional appearance.

As to the testicle, the findings varied. Slight or pronounced enlargement of the testicle was found in six cases. All animals save one, which died during the treatment, showed a definite enlargement of the seminal vesicles. The histological findings differed considerably. In four animals spermatogenesis, which was found to be absent in the control testicle removed before the injections started, had recurred in the remaining testicle. This result cannot be easily reconciled with our general knowledge regarding the influence of gonadotropic hormones upon the testicle, since experiments carried out in this laboratory and elsewhere have failed to establish spermatogenesis as an effect of gonadotropic hormones. In the remaining cases the histogenetic influence of the injections upon the testicle was slight and apparently restricted to the interstitial tissue ; this is in accordance with findings on prepuberal or pubescent animals. The influence of the injections upon the general condition of the animals was not marked, although it seems that in a few cases an improvement in the general condition occurred; in the remaining animals no distinct influence was manifest.

The experiments so far conducted show, in accordance with previous findings, that the senile gonad can be reactivated, at least in some cases. The senescence of the gonad seems to be due to the lack of the specific stimulating factor represented by the gonadotropic hormones of the anterior lobe, and not to any general condition of the organism or to the absolute age of the gonad itself. It is difficult to reconcile with these conclusions observations which claim that interference with the testicle itself led to a reactivation of testicular secretion, since the deficiency is one of extra-gonadic factors. However, further experiments with vaso-ligature and similar methods are in progress. The experiments with injections of gonadotropic extracts are also repeated on a larger scale so that any existing influence on duration of life should be detected.

\section{Growth and Senility}

In view of the fact that no absolute correlation between gonadic function and the senescence of the organism as a whole was manifest, it seemed desirable to examine the relation between general functions and senescence. The investigation of growth in relation to senescence suggests itself. Growth may be regarded not as an incidental attribute of the early phases of life, but as an essential condition of what, for the present, one might call " youth," whether- the latter be defined statistically (expectation of life) or biologically (by reference to the intensity of functions, efficiency of the organism as a whole, etc.). ${ }^{1}$

Experiments with the injection of growth-promoting extracts from the anterior lobe into aged animals were therefore arranged. The extracts used were either pre-

1 To regard growth as more than a mere epiphenomenon of youth seems justifiable from a general biological point of view ; the classical experiments of Hartmann on the immortalization of Planaria may be mentioned in this context, and particular reference may also be made to the point of view developed by Ehrenberg, according to whom the term "growth" may be taken in a wider sense than the usual one which implies actual increase in size. pared by the ammonia method (Wiesner and Sheard) and contained gonadotropic hormones or else were free of the latter, the method of van Dyke and his colleagues being used. So far the experiments have shown that many senile animals can react with considerable growth to the administration of growth-promoting extracts. In the meantime the increase in weight is taken as the sole indicator of growth. This is permissible in the absence of pathological conditions such as anasarca or obesity; any error introduced by the use of the weight curve as an indicator of general growth will, it is hoped, be corrected by $x$-ray records to be taken in future experiments.

It appears that in some cases the administration of growth extracts had a beneficial influence upon the general condition of the rat. At present the mortality rate of the experimental male group is only half that of the uninjected special control group, while no significant differences between respective mortality rates of the experimental and control females have occurred. Yet it will remain impossible to draw any definite and reliable conclusion before the groups will have died off.

It need hardly be emphasized that the observations briefly related above represent only a few preliminary notes. Our observations, however, may serve as indicating lines of further research. It is hoped that by the end of the coming year the number of experiments on aged animals will be sufficiently large to permit more definite conclusions. Also, experiments undertaken by the writer's colleagues will probably yield some more objective data as to changes occurring in old age and the reaction of the senile organism to the administration of gonadotropic and growth-promoting extracts.

The writer would like to express his gratitude to Miss N. M. Sheard, who is superintending the rat colony, and to Mr. T. B. Macaulay, LL.D., whose generosity made it possible to build up and maintain the colony.

\section{ANEURYSM OF ABDOMINAL AORTA AND THROMBOSIS OF SUPERIOR MESENTERIC ARTERY ASSOCIATED WITH A BULLET WOUND OF THE LUNG}

BY

J. GILMOUR, M.B., M.S., F.R.C.S. HONORARY ASSISTANT SURGEON

AND

STUART MCDONALD, JUn., M.A., M.B., M.R.C.S. ASSISTANT PATHOLOGIST, ROYAL VICTORIA INFIRMARY, NEWCASTLE-ON-T THSE

The case recorded below has considerable medico-legal interest in respect to the establishment of a causal relation between the passage of the bullet and the formation of the aneurysm. There is no evidence of syphilis as an aetiological factor, neither does the site of the aneurysm nor its form correspond with those of an atheromatous lesion. In any case the abdominal aorta is a rare site for spontaneous aneurysms, and since the lesion present corresponds in type to a traumatic aneurysm, one is forced to the conclusion that the latter diagnosis is correct.

\section{CAse Record}

The patient, an ex-soldier aged 45, was admitted to the Royal Victoria Infirmary, Newcastle-on-Tyne, at 1.30 p.m. on July 24th, 1931, suffering from an " acute abdominal crisis " of thirty-six hours' duration, with a diagnosis of intussusception. $\mathrm{He}$ had been taken ill thirty-six hours previously with pain in the epigastrium, nausea, and vomiting, and had been treated medically for gastro-enteritis. The bowels had been opened several times during the attack and 
blood had been passed in the stools. On admission, the patient was profoundly collapsed and was found to be suffering from general peritonitis, but there was nothing definitely pointing to intussusception. His condition precluded operative interference, and he sank rapidly and died nine hours after admission.

\section{Previous History}

This was obtained after death from hospital records, etc. On February 2nd, 1916, the patient was on active service in France and was struck by a bullet in the epigastric region. $\mathrm{He}$ remained for five days in a C.C.S. During this time he was too ill to be evacuated. "His abdomen was very swollen and he could only take sips of water." $\mathrm{He}$ was later transferred to the Norfolk War Hospital, where he remained for thirty-nine days. The records show that he suffered from a G.S.W. of chest with entry to the left of mid-line over the sternal end of seventh rib. $X$-ray examination showed a bullet lying between the tenth and eleventh ribs on the right side, close to the spine. There were no symptoms or signs of pulmonary disease. He was eventually discharged to light duty. Between 1916 and 1921 he came before various Medical Boards complaining of dyspnoea and pain in the chest, but no evidence of pulmonary disease could be found. His incapacity assessment was 30 per cent. At an examination on February 10th, 1921, breath sounds were found to be diminished over the right base and there was increased tactile vocal fremitus and vocal resonance on the right side. A year later he still complained of cough, dyspnoea, and of being easily fatigued, and although physical examination proved negative the Board was of opinion that the diaphragm had been injured and that it was this injury which was causing the symptoms. In March, 1923, the same complaints were still present but physical signs were equivocal. An $x$-ray examination showed the bullet clear above the diaphragm, the shadow being seen in the lateral view, moving with the diaphragm. The depth from the front was 3 to 4 inches and from the back $2 \frac{1}{2}$ to 3 inches. There was possible injury to the eleventh dorsal vertebra (the final opinion of the Ministry of Pensions was that the more accurate description of the bullet's level would be between the eleventh and twelfth dorsal vertebrae).

The only other points of importance in the patient's medical history were hypertension, with accentuation of the second aortic sound and slight albuminuria (1924), abscess in arm (1924), renal colic with haematuria and passage of calculus per urethram (1928), and "cold" and neuritis complicated by pyorrhoea (1930).

\section{Pathological Investigation}

A necropsy was performed on July 27 th, 1931, at the request of the coroner. The following is an abstract of the findings:

No scar was visible in the left epigastric region. There was no pericarditis. Beyond some dilatation of the right auricle, slight hypertrophy of the left ventricle, slight nodular atheroma of the first part of the aorta and coronary arteries, and toxic changes in the myocardium, the heart showed nothing abnormal. The right pleural cavity was obliterated by dense adhesions, and the right cupola of the diaphragm was adherent to the lung base. Midway between the anterior and posterior borders of the lung at the base and immediately beneath the pleura a bullet was found embedded in the substance of the lung, with the nose pointing in an antero-lateral direction. On section, the lung showed terminal passive congestion and oedema. There was no consolidation. The left pleural cavity and.left lung appeared healthy save for terminal passive congestion and oedema at the lung base.

A considerable amount of blood-stained effusion was present in the peritoneal cavity. The stomach and intestines were distended with gas and fluid. The coils of small intestine and the right half of the transverse colon showed marked congestion and subperitoneal haemorrhage, with a fibrinous exudate on the surface. In places, the small intestine was plum-coloured. Extending between the origin of the superior mesenteric artery and the right crus of the diaphragm was a saccular aneurysm of the aorta about the size of a walnut. The under surface of the sac was densely adherent to the tissues anterior to the lumbar vertebrae, there being much scar tissue present in this region. On opening the acrta the orifice of the sac was found to be $1 \mathrm{~cm}$. in diameter, and was situated on the posterior wall of the aorta towards the right side. The lips of the orifice consisted of dense scar tissue. On dissection of the superior mesenteric artery its lumen was found to be occluded by ante-mortem thrombus. The other branches of the coeliac axis and the inferior mesenteric artery were healthy, as was the inferior vena cava and its main tributaries. The intima of the aorta showed a moderate amount of nodular atheroma, but there was no gross evidence of syphilis. At the bifurcation of the aorta a large antemortem thrombus was found, which extended into the common iliac arteries as far as their bifurcation. The spleen, pancreas, liver, suprarenals, and right kidney showed only changes attributable to toxaemia and post-mortem change. The gall-bladder and bile ducts were healthy. The left kidney was enlarged and showed advanced simple hydronephrosis, the structure corresponding to dilated calyces associated with a thin rim of cortical tissue. The calyces contained clear urine and a few small calculi. The left ureter was thickened and dilated, and a medium-sized calculus was impacted at its lower end, completely blocking the lumen. The right ureter, urinary bladder, and prostate showed nothing abnormal. There was no evidence of injury or disease in the skull or brain.

Anatomical Diagnosis.-Lodgement of bullet in right lung, aneurysm of abdominal aorta, thrombosis of superior mesenteric and common iliac arteries, infarction of small intestine, calculous hydronephrosis.

Histological examination of the following tissues was carried out.

Heart.-There was no evidence of acute or chronic inflammatory change. The circumflex branch of the left coronary artery showed slight musculo-elastic medial hyperplasia and some intimal thickening.

First Part of Aorta.-There was no thickening of adventitia or media and no endarteritis or perivascular cuffing of the vasa vasorum. There was no fibrosis of the media and a normal amount of elastic tissue was present. The intima showed slight atheroma.

Anerrysmal Sac.-There were small collections of lymphocytes in the adventitial region. The wall of the sac consisted of mature collagen with a fair amount of elastic tissue. There was no vascularization or cellular infiltration. No trace of intimal tissue was seen. The contents of the sac consisted of ante-mortem thrombus without evidence of organization, and some post-mortem coagulum.

Right Common Iliac Artery.-There was some fibrous thickening of adventitia and media with atheromatous change in the intima. The lumen contained mixed thrombus without evidence of organization.

Base of Right Lung.-The pleura was grossly thickened, the appearances being those of mature scar tissue in which a few large vessels and small collections of lymphocytes were present. The underlying lung showed areas of oedema and collapse alternating with emphysematous areas. The larger vessels showed some endarteritis and the alveolar capillaries were congested. There was considerable hyperplasia of the fibrous stroma of the lung, and at the site of the bullet mature scar tissue and foci of histiocytes were present.

Right Kidney.-Apart from toxic and post-necrotic changes and the occurrence of a few fibrosed glomeruli, the renal tissue was healthy. There was a slight degree of arteriosclerotic thickening of the main branches of the renal artery.

Left Kidney.-This showed similar appearances except in the neighbourhood of the distended calyces, where there was marked glomerular and tubular atrophy associated with diffuse fibrosis and large foci of histiocytes. The transitional epithelium of the calyces had been replaced by scar tissue.

\section{Discussion}

Since a period of fifteen years elapsed between the original wound and death, accurate determination of the exact course of the bullet must be largely a matter of conjecture, for reparative processes might reasonably be expected to 
be very complete. We are of opinion, however, that the bullet took a downward and backward course after its entry and passed below the diaphragm, after which it struck the spinal column, and passed through the diaphragm on the right side. The symptoms from which the patient suffered while at the C.C.S. might be interpreted as being due to injury to the liver and haemothorax, and although no positive evidence as to the former was found at necropsy, the pleural adhesions present on the right side might be attributable to spontaneous cure of the latter condition. The various findings of the Boards certainly suggest diaphragmatic injury, and with regard to the $x$-ray appearances the site of the bullet between the eleventh and twelfth dorsal vertebrae, with possible injury of the eleventh dorsal vertebra, affords corroborative evidence of our suggestion as to the path of the bullet. Moreover, since the aneurysmal sac extended between the origin of the superior mesenteric artery and the under surface of the diaphragm on the right side, its site corresponds to the lower half of the twelfth and the upper half of the first lumbar vertebra. This point is sufficiently near the final position of the bullet (in the right lung base immediately above the diaphragm) to indicate an association between the path of the bullet and the aneurysm. As to the exact nature of the injury which the aortic wall must have sustained, one is left in considerable doubt, and it is remarkable that in the circumstances damage to either aorta or inferior vena cava did not result in rapid death. Since this did not happen, the formation of an aneurysm may have been due to: (1) grazing injury to the aortic wall such as might be produced by $(a)$ the passage of a bullet, (b) a dislodged spicule of bone ; or (2) inflammatory reaction in the track of the bullet. The combination of cicatricial weakening from the injury and the intra-aortic pressure would necessarily lead to a progressive local dilatation of the aorta.

We sent the bullet to Professor Sydney Smith of the Forensic Medicine Department of Edinburgh University, to whom we are indebted for the following report:

"It is a Danish bullet composed of a lead core with steel and nickelled casing. It was fired from a German rifle. It was not a direct hit but was a ricochet, having struck a hard surface before it hit the victim. This would give it a more eccentric course and would explain its direction in the body. After entering in the region of the seventh rib to the left of the middle line, it no doubt struck the aorta a glancing blow, which caused a certain amount of bruising; then passed between the pillars of the diaphragm into the chest cavity. The damage to the wall of the aorta would lead to fibrosis and stretching."

We are unable to account for the clinical latency of the condition with regard to dislodgement of portions of thrombus from the sac, which might have occurred at any time and ultimately caused death. The condition of calculous hydronephrosis, which was present, was apparently an entirely separate pathological process, and can be associated neither with the bullet wound nor with the final illness.

\section{OBSERVATIONS ON THE TREATMENT OF TETANUS}

\section{(WITH SPECIAL REFERENCE TO TETANUS ANTITOXIN)*} BY

\section{B. B. YODH, M.B., M.R.C.P., D.T.M. AND H. HONORARY PHYSICIAN, J. J. HOSPITAL, BOMBAY}

My purpose in dealing with this subject is not to describe any new method of treatment or to claim any startling results, but to take stock of the present situation regarding the various methods of treatment advocated and of the methods tried in this group of cases. A continuous charge of the male tetanus wards for five years has enabled me to observe a fairly large number of cases, and to vary, and perhaps improve, the technique of treatment from time to time.

\section{Methods of General Treatment}

The general treatment is very important, and I propose to deal with this first.

The fatal effects of tetanus are principally due to: (1) poisoning of the vital centres, causing respiratory and cardiac failure; (2) exhaustion due to continuous muscular spasm and repeated seizures on the top of it, the latter being intensely aggravated by peripheral stimuli, even very light ones. To minimize this second factor requires very careful, painstaking, and intelligent nursing.

A well-ventilated dark room is very important, as by this means the external stimuli are considerably reduced. In the construction of small tetanus wards the walls should be painted dark, window shutters obliquely placed, and an exhaust fan provided. There should be silence in the ward. The slightest noises, such as caused by shuffling of the feet or jingling of glass, bring on spasms. Bromides, chloretone, chloral hydrate, hyoscyamus, etc., are the usual sedatives employed. Large doses are required at the beginning. Morphine with atropine may be useful when the spasms are very severe, and during the early stages.

* Being the Lord Reay Lectures delivered at the Grant Medical College in November, 1931.
Too much morphine, however, should be avoided, especially when pulmonary complications are present. 'The prolonged use of these sedatives is attended with some danger, and one has come across several cases of drowsiness and even coma after the spasms.have passed awayprobably as a cumulative effect of the sedatives.

\section{、 Chloroform and Special Drugs}

Although all the textbooks prescribe it, and in some French journals a few cases have been reported in which enormous doses have been used with apparently good results, I do not advocate the use of chloroform for the relief of spasms. Our own experience of chloroform, which was used in earlier cases, is not satisfactory, as pulmonary oedema supervened in a fair number of these cases with fatal results. It is, of course, difficult to prove that chloroform was the factor concerned, but such is the clinical impression. We now avoid chloroform even while performing cistern or lumbar puncture. Anaesthesia, which may be required in some cases for the extreme rigidity and frequent spasms, can easily be obtained by the use of liq. somniferus (Stella and Co.). This is quite safe and gives immediate unconsciousness which acts for a short time only. It has been used in about a dozen instances in this series, and no ill effects have been observed.

Injection of special drugs, such as carbolic acid and magnesium sulphate, has been recommended for the relief of spasms, but they have not been used in this series of cases at all. On looking up the recent literature one does not find any great claims made for them, although the older textbooks recommend them. Recently two articles have been published regarding the carbolic acid treatment (Bacelli) of tetanus. Pilloni ${ }^{11}$ has treated fiftythree cases over a period of twelve years, with 70 per cent. cures, by injection of phenol 0.5 gram for the first two days, followed by smaller doses. He had only three cases of nephritis in the series. Suvansu ${ }^{12}$ has treated fourteen consecutive cases of tetanus with intrathecal injections of carbolic acid, with four deaths, and has not 